\title{
Protective effects of anti-C5a in sepsis-induced thymocyte apoptosis
}

\author{
Ren-Feng Guo, ${ }^{1}$ Markus Huber-Lang, ${ }^{1}$ Xin Wang, ${ }^{1}$ Vidya Sarma, ${ }^{1}$ \\ Vaishalee A. Padgaonkar, ${ }^{1}$ Ronald A. Craig, ${ }^{1}$ Niels C. Riedemann, ${ }^{1}$ \\ Shannon D. McClintock, ${ }^{1}$ Tommy Hlaing, ${ }^{1}$ Michael M. Shi, ${ }^{1,2}$ and Peter A. Ward ${ }^{1}$
}

${ }^{1}$ Department of Pathology, University of Michigan Medical School, Ann Arbor, Michigan, USA

${ }^{2}$ Department of Drug Safety Evaluation, Pfizer Global Research and Development, Ann Arbor, Michigan, USA

Address correspondence to: Peter A. Ward, Department of Pathology, University of Michigan Medical School, 1301 Catherine Road, Ann Arbor, Michigan 48109-0602, USA.

Phone: (734) 763-6384; Fax: (734) 763-4782; E-mail: pward@umich.edu.

Received for publication July 14, 2000, and accepted in revised form September 29, 2000.

\begin{abstract}
Multiorgan apoptosis occurs during sepsis. Following cecal ligation and puncture (CLP) in rats, thymocytes underwent apoptosis in a time-dependent manner. C5a blockade dramatically reduced thymocyte apoptosis as measured by thymic weight, binding of annexin $\mathrm{V}$ to thymocytes, and laddering of thymocyte DNA. When C5a was generated in vivo by infusion of purified cobra venom factor (CVF), thymocyte apoptosis was significantly increased. Similar results were found when CVF was injected in vivo during the early stages of CLP. In animals 12 hours after induction of CLP, there was an increase in the activities of caspase-3, -6 , and -9 , but not caspase-1 and -8. Cytosolic cytochrome $c$ levels increased by twofold, whereas mitochondrial levels showed a $50 \%$ decrease. Western blot analysis revealed that the content of Bcl- $X_{\mathrm{L}}$ (but not of Bcl-2, BAX, Bad, and Bim) significantly decreased in thymocytes after CLP. C5a blockade in the sepsis model almost completely inhibited caspase- $3,-6$, and -9 activation, significantly preserved cytochrome $c$ in the mitochondrial fraction, and restored $\mathrm{Bcl}-\mathrm{X}_{\mathrm{L}}$ expression. These data suggest that systemic activation of complement induces $\mathrm{C} 5 \mathrm{a}$-dependent apoptosis of thymocytes and that the blockade of $\mathrm{C} 5 \mathrm{a}$ during sepsis rescues thymocytes from apoptosis.
\end{abstract}

J. Clin. Invest. 106:1271-1280 (2000).

\section{Introduction}

Sepsis, together with systemic inflammatory response syndrome (SIRS) and consequent multiple organ failure syndrome (MOFS), is the most common cause of death in intensive care units (1). Increasing evidence shows that apoptosis occurs in many organs during sepsis. In experimental models of sepsis, apoptosis was noted in the thymus, spleen, Peyer's patches, liver, kidney, lung, intestine, and skeletal muscle (2-4). In patients with sepsis, focal apoptosis in the spleen, colon, and ileum has been demonstrated (5). The onset of apoptosis seems to accompany the development of MOFS in sepsis. Many cell types are the targets for apoptosis during sepsis. Sepsis delays neutrophil apoptosis but increases apoptosis in T-lymphocytes, B-lymphocytes, macrophages, epithelial cells, and endothelial cells (2-4). Lymphocytes appear to be the predominant cell target for apoptosis during sepsis. Extensive lymphocyte apoptosis accompanied by depletion of lymphocytes in white pulp of the spleen and development of lymphocytopenia has been found in most cases of humans with sepsis (5). Atrophy of the thymus associated with thymocyte apoptosis has been observed in a model of Gram-negative or -positive sepsis, as well as in CLP-induced sepsis in rodents (6-8). Tcell suppression and a decrease in total T-lymphocyte numbers are characteristic symptoms in MOFS (2).
Transgenic mice that selectively overexpress Bcl-2 in T cells showed almost complete protection against $\mathrm{T}$ lymphocyte apoptosis and had improved survival in sepsis (9). Administration of $z$-VAD-fmk, a pan-caspase inhibitor, blocked lymphocyte apoptosis in thymocytes and splenocytes and resulted in marked improvement in survival in CLP-induced sepsis in mice (9). These data suggest that widespread lymphocyte depletion induced by apoptosis may be attributable to immunosuppression that occurs in sepsis.

Activation of the alternative pathway of complement is considered to be an effective defense mechanism against invading microorganisms. However, excessive activation may lead to compromised host defenses (10). There is strong evidence for complement activation in sepsis. Activation of complement as reflected by elevated plasma levels of anaphylatoxins, C3a and C5a, has been documented in the baboon and rat septic shock models $(11,12)$. There is also clear evidence for presence of complement activation products, $\mathrm{C} 3 \mathrm{a}, \mathrm{C} 4 \mathrm{a}$, and $\mathrm{C} 5 \mathrm{a}$, in the plasma of septic humans $(13,14)$. Complement is known to contribute to regulation of the immune response (15). C5a is considered to be one of the most potent inflammatory peptides with many biologic functions (16). In vivo blockade of C5a significantly improves survival rates in experimental sepsis $(12,17,18)$. However, little is known concerning the mechanism underlying 
the protective effects of C5a blockade in sepsis. In the current studies, we sought to determine whether blockade of C5a would affect thymocyte apoptosis in rats undergoing CLP-induced sepsis in an attempt to understand potential roles of $\mathrm{C} 5 \mathrm{a}$ in immunoregulation during sepsis. We also induced in vivo generation of $\mathrm{C} 5 \mathrm{a}$ by intravenous infusion of CVF, to determine whether there was an increase in C5a-dependent apoptosis.

\section{Methods}

Experimental sepsis induced by CLP. Male Long-Evans specific pathogen-free rats (8-12 weeks old; Harlan Inc., Indianapolis, Indiana, USA) were used in all studies. Anesthesia was induced by intraperitoneal administration of ketamine (20 mg/100 mg body weight). Through a $2-\mathrm{cm}$ abdominal midline incision, the cecum was ligated below the ileocecal valve without obstruction the ileum or colon. The cecum was then subjected to a single "through and through" perforation with a 21-gauge needle. After repositioning the bowel, the abdominal incision was closed in layers with plain gut surgical suture 4-0 (Ethicon Inc., Somerville, New Jersey, USA) and metallic clips. Sham animals underwent the same procedure except for ligation and puncture of the cecum. At different time points, the rats were sacrificed, and the thymus was removed and weighed. Thymocytes were dissociated from thymus in nontreated, sham, or CLP rats according to Morris et al. (19) with minor modifications.

For antibody intervention, immediately after induction of sepsis, some animals received either $400 \mu \mathrm{g}$ rabbit anti-rat C5a intravenously or $400 \mu \mathrm{g}$ preimmune rabbit IgG. Preparation and characterization of antirat C5a are described elsewhere (18). This polyclonal antibody does not block whole complement hemolytic activity (CH50) in rat serum (data not shown). Immunoprecipitation with activated rat serum using beads coupled with this antibody yielded a characteristic rat $\mathrm{C} 5 \mathrm{a}$ protein band in the $14-\mathrm{kDa}$ position in Western blot analysis (20).

Complement activation in vivo. Cobra venom factor (CVF) was purified from lyophilized cobra venom (Naja naja kaouthia) (Sigma Chemical Co., St. Louis, Missouri, USA) by ion exchange chromatography and gel filtration (10). Three units of CVF were injected intravenously into normal male 275- to 300 -g LongEvans rats or into rats 3 hours after induction of CLP. Three hours after injection of CVF, animals were sacrificed, and thymocytes were isolated as already described here and analyzed for evidence of apoptosis.

Analysis of DNA fragmentation. Thymocytes $\left(1 \times 10^{7}\right)$ were lysed in lysis buffer ( $6 \mathrm{M}$ guanidine- $\mathrm{HCl}, 10 \mathrm{mM}$ urea, $10 \mathrm{mM}$ Tris- $\mathrm{HCl}$, and 20\% Triton X-100 [pH 4.4]). The DNA was extracted by a DNA purification kit (Boehringer-Mannheim Corp., Mannheim, Germany). After quantification, $2 \mu \mathrm{g}$ of each DNA sample was subjected to electrophoresis on a $1.5 \%$ agarose gel. The gels were stained with ethidium bromide $(10 \mu \mathrm{g} / \mathrm{ml})$, and were photographed under ultraviolet illumination.
Detection of thymocyte apoptosis by flow cytometry analysis. Thymocytes $\left(2 \times 10^{6}\right)$ were stained with Annexin V (Ax) conjugated to FITC and propidium iodide (PI) by using the Annexin-V-FLUOS staining kit (BoehringerMannheim Corp.) according to the manufacturer's instruction. Immediately after staining, the cells were analyzed on a flow cytometer (Coulter Corp., Miami, Florida, USA) using $488 \mathrm{~nm}$ excitation and a $525-\mathrm{nm}$ bandpass filter for FITC and a 620-nm filter for PI detection. Data were analyzed by using WinList computer software (Verity Software House Inc., Topsham, Maine, USA). Electronic compensation of instrument was carried out to exclude overlapping of the two emission spectra. Cell debris was eliminated by gating according to side-scatter and forward-scatter detection. The regions for Ax-positive only, PI-positive only, double-positive, and double-negative were created by single-parameter analysis with Ax-FITC or PI.

Caspase activity assay. Caspase- $1,-3,-6,-8,-9$ activities were determined by a fluorometric assay (Santa Cruz Biotechnology Inc., Santa Cruz, California, USA) according to the manufacturer's instructions. Briefly, cell lysate from $1 \times 10^{6}$ thymocytes was incubated with caspase substrates (50 $\mu \mathrm{M}$ final concentration): AcYVAD-AFC (caspase-1), Ac-DEVD-AFC (caspase-3), AcVEID-AFC (caspase-6), Ac-IETD-AFC (caspase-8), and Ac-LEHD-AFC (caspase-9). Two hours after incubation at $37^{\circ} \mathrm{C}$, any cleaved AFC was detected on a Cytofluor II plate reader (Millipore Corp., Bedford, Massachusetts, USA) with a 400-nm excitation and 505-nm emission filter. Fluorescent units were converted to picomoles of AFC using a standard curve of free AFC. The results were expressed as picomoles of AFC released from substrates per microgram protein over a 2-hour incubation time.

Determination of cytosolic and mitochondrial cytochromec content. Subcellular fractionation was extracted as described previously with minor modifications (21). Briefly, cells were lysed in lysis buffer (10 mM EDTA, $5 \mathrm{mM}$ EGTA, $20 \mathrm{mM}$ Tris-HCl [pH 7.4], and protease inhibitor), and homogenized in Dounce homogenizer by 40 strokes on ice. The lysates were centrifuged for 10 minutes at $900 \mathrm{~g}$, and then the supernatant was centrifuged for 10 minutes at 5,000 $\mathrm{g}$. The pellet fraction (mitochondria) was solubilized in PBS containing $0.5 \%$ Triton $\mathrm{X}-100$. The supernatant was recentrifuged at $100,000 \mathrm{~g}\left(4^{\circ} \mathrm{C}\right.$ for 45 minutes) to provide the cytosol fraction. Cytochrome $c$ concentrations in cytosol and mitochondria were quantitatively determined using a cytochrome $c$ ELISA kit (R\&D Systems Inc., Minneapolis, Minnesota, USA) according to the manufacturer's protocol.

Western blot analysis. Cells were lysed in the lysis buffer and homogenized in Dounce homogenizer with 40 strokes on ice. The cytoplasmic extracts were obtained by centrifuging the lysates for 10 minutes at $900 \mathrm{~g}$. Protein levels were determined by a Bio-Rad Protein Assay (Bio-Rad Laboratories Inc., Hercules, California, USA). Samples containing $100 \mu \mathrm{g}$ protein were elec- 


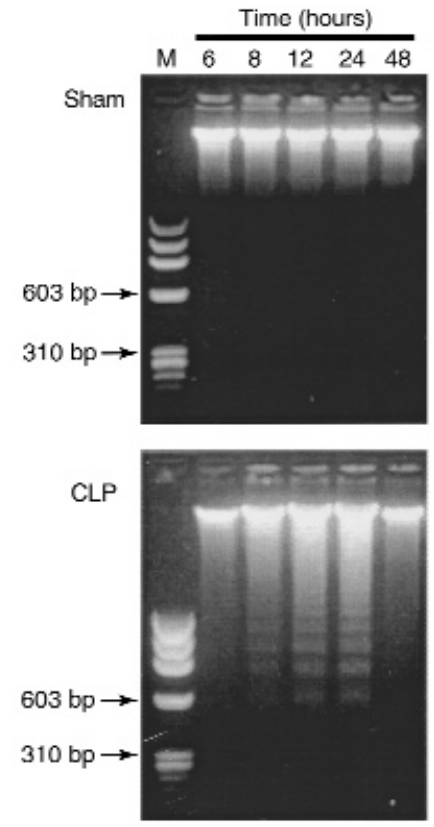

\section{Figure 1}

Time course of CLP-induced DNA fragmentation in thymocytes. DNA was extracted from thymocytes of sham or CLP rats at indicated hours and was subjected to agarose gel electrophoresis. M, DNA molecular weight marker. The results are representative of three separate experiments.

trophoresized in a denaturing $10 \%$ polyacrylamide gel and then transferred to a nitrocellulose membrane. Nonspecific binding sites were blocked with Trisbuffered Saline-Tween (TBST) (40 mM Tris [pH 7.6], $300 \mathrm{mM} \mathrm{NaCl}$, and $0.1 \%$ Tween 20 ) containing $5 \%$ nonfat dry milk for 12 hours at $4^{\circ} \mathrm{C}$. Membranes were incubated with the following antibodies in a 1:1,000 or 1:2,000 dilution: polyclonal rabbit anti-Bcl-2 (PharMingen, San Diego, California, USA), polyclonal rabbit anti-BAX (PharMingen), monoclonal mouse anti-Bcl- $\mathrm{X}_{\mathrm{L}}$ (PharMingen), polyclonal rabbit anti-Bad (Santa Cruz Biotechnology Inc.), polyclonal rabbit anti-Bim (PharMingen), and monoclonal mouse anti- $\alpha$-tubulin (Sigma Chemical Co.). After five washes in TBST, membranes were incubated in a 1:10,000 dilution of horseradish peroxidase-conjugated donkey anti-rabbit or mouse IgG (Amersham Pharmacia Biotech, Piscataway, New Jersey, USA). The membrane was developed by enhanced chemiluminescence technique according to the manufacturer's protocol. Protein expression was quantitated from digitized autoradiography films using image analysis software (Adobe Systems, San Jose, California, USA). Protein levels were normalized by probing the same blot with $\alpha$-tubulin.

Assessment of NF- $\kappa B$ activation by electrophoretic mobility shift assay. Nuclear extracts were prepared using the method described previously (22). Protein concentrations were determined by bicinchoninic acid assay with trichloroacetic acid precipitation using BSA as a reference standard (Pierce Chemical Co., Rockford, Illinois, USA). Double-stranded NF- $\mathrm{KB}$ consensus oligonucleotide (5'-AGTGAGGGGACTTTCCCAGGC-3'; Promega Corp., Madison, Wisconsin, USA) was endlabeled with $\gamma\left[{ }^{32} \mathrm{P}\right]$ ATP $(3,000 \mathrm{Ci} / \mathrm{mmol}$ at $10 \mathrm{mCi} / \mathrm{ml}$; Amersham Pharmacia Biotech). Binding reactions containing $5 \mu \mathrm{g}$ nuclear extracts and 35 fmols $(\sim 50,000$ $\mathrm{cpm}$, Cherenkov counting) oligonucleotide were performed for 30 minutes in binding buffer [ $4 \%$ glycerol, $1 \mathrm{mM} \mathrm{MgCl}_{2}, 0.5 \mathrm{mM}$ EDTA ( $\mathrm{pH} 8.0$ ), $0.5 \mathrm{mM}$ dithiothreitol, $50 \mathrm{mM} \mathrm{NaCl}, 10 \mathrm{mM}$ Tris ( $\mathrm{pH}$ 7.6), and 50 $\mu \mathrm{g} / \mathrm{ml}$ poly $(\mathrm{dI} \bullet \mathrm{dC})$; Amersham Pharmacia Biotech].

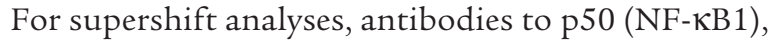
p52 (NF-кB2), p65 (RelA), p68 (RelB), or p75 (c-Rel) were added 15 minutes before adding the radiolabeled NF-KB oligonucleotide. All of these antibodies used in the electrophoretic mobility shift assay (EMSA) experiments were from Santa Cruz Biotechnology Inc. Reaction volumes were held constant to $15 \mu \mathrm{l}$. Reaction products were separated in a $4 \%$ polyacrylamide gel and analyzed by autoradiography. NF- $\mathrm{\kappa B}$ activation was quantitated from digitized autoradiography as stated previously.

\section{Results}

Sepsis induces thymocyte apoptosis in a time-dependent manner. Although thymocyte apoptosis has been extensively studied, the time course of thymocyte apoptosis during sepsis in CLP rats has not been well addressed. In this study, during the time course of 48 hours after sham or CLP induction, thymocyte apoptosis induced by sepsis was monitored by DNA fragmentation and FACS analysis. DNA isolated from thymocytes of sham rats did not demonstrate appreciable fragmentation (Figure 1). DNA fragments with multiples of 180-200 bp, a hallmark of apoptotic cell death, were identifiable in thymocytes 8 hours after induction of CLP. The ladderlike DNA fragments were very distinct at 12 and 24 hours, disappearing at 48 hours (Figure 1).

The time dependency of thymocyte apoptosis in sepsis was also manifested by analysis of phosphatidylserine (PS), an early apoptotic marker on the outer leaflet of apoptotic cell membranes. At different time points after sham or CLP surgery, thymocytes were isolated, stained with Ax-FITC and PI, and analyzed on a flow cytometer. Four groups of cells were differentiated according to the staining patterns: normal cells $\left(\mathrm{Ax}^{-} \mathrm{PI}^{-}\right)$, early stages of apoptotic cells $\left(\mathrm{Ax}^{+} \mathrm{PI}^{-}\right)$, late apoptotic cells and early necrotic cells $\left(\mathrm{Ax}^{+} \mathrm{PI}^{+}\right)$, and necrotic cells $\left(\mathrm{Ax}^{-} \mathrm{PI}^{+}\right)$(Figure 2a). Constitutive early-stage apoptosis was observed with a percentage of $3-5 \%$ in the whole thymocyte population (time 0 , Figure $2 b$ ). Sham surgery resulted in a small amount of early-stage apoptosis $(<10 \%)$. However, the percentage of early-stage apoptotic cells started to increase 6 hours after the onset of sepsis, reaching approximately $30 \%$ at 12 and 24 hours, and then decreasing to approximately $15 \%$ at 48 hours (Figure 
$2 b)$. CLP also induced a progressive increase in the percentage of late apoptotic and early necrotic thymocytes, but sham surgery did not (Figure 2c).

Reduced thymus weight loss and thymocyte apoptosis in sepsis after blockade of $\mathrm{C} 5 \mathrm{a}$. Thymus weight loss after onset of sepsis was demonstrated dramatically (Figure 3). Significant weight loss occurred as early as 12 hours after CLP surgery $(P<0.05)$. Thymus weights reached their lowest levels (at $50 \%$ ) by 24 hours $(P<0.01)$. No further weight loss was found at 48 hours. C5a blockade dramatically reduced the thymic weight loss caused by CLP over 48 hours. In CLP rats treated with anti-C5a, thymus weights were not significantly changed within 24 hours (Figure 3 ). The thymus weight slightly decreased at 48 hours in the anti-C5a-treated group, but was significantly higher than those in the CLP group $(P<0.01)$.

The effect of C5a blockade on apoptosis as determined by DNA fragmentation was evaluated 24 hours after onset of sepsis. CLP-induced DNA fragmentation was reduced below detectable levels after blockade of C5a (Figure 4a). Correspondingly anti-C5a treatment resulted in a significant decrease in CLP-induced earlystage apoptotic cells by $80 \%(P<0.01)$ (Figure 4 , b and $c)$, whereas late apoptotic and early necrotic cells decreased by $93 \%(P<0.05)$ in CLP animals treated with anti-C5a (Figure 4d).
Inbibitory effects of C5a blockade on caspase activation and cytochrome c release from mitochondria of thymocytes during sepsis. Based on the time course of thymocyte apoptosis in sepsis (Figure 2b), a time point of 12 hours was used to evaluate caspase activation and cytochrome $c$ translocation from mitochondria to the cytosol. In comparison to sham controls, caspase-3 activity in thymocytes increased threefold, and caspase- 6 and caspase- 9 activity elevated twofold 12 hours after CLP. Caspase- 1 activity was not detectable, and caspase-8 activity was not significantly affected (Figure 5). C5a blockade almost completely inhibited caspase-3, -6 , and -9 activation occurring after CLP $(P<0.05)$ (Figure 5).

In thymocytes from CLP rats, there was a significant increase ( twofold) in the amount of cytochrome $c$ present in the cytosol and a concordant decrease $(\sim 50 \%)$ of cytochrome $c$ in the mitochondria fraction when compared with sham controls (Figure 6, a and b). After anti-C5a treatment, the concentration of cytochrome $c$ dropped by $68 \%$ in the cytosolic fraction when compared with CLP rats treated with preimmune IgG and was maintained at $91 \%$ of the reference control level in the mitochondrial fraction $(P<0.05)$ (Figure 6).

Effects of C5a blockade on Bcl-2, Bcl- $X_{L}, B A X$, Bad, and Bim expression in thymocytes. Thymocytes from normal, sham, and CLP- and anti-C5a-treated CLP rats were

a

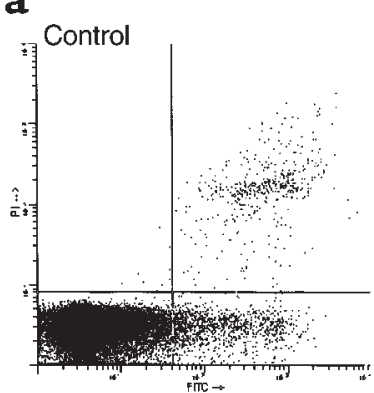

b

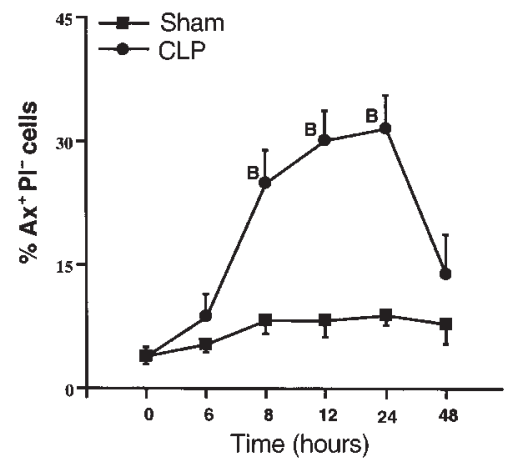

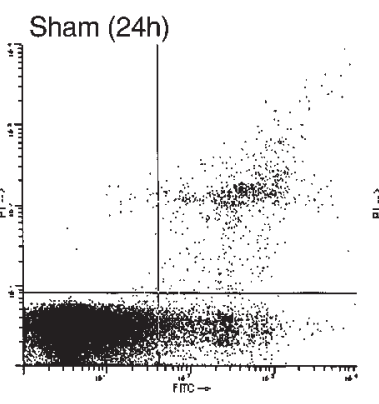

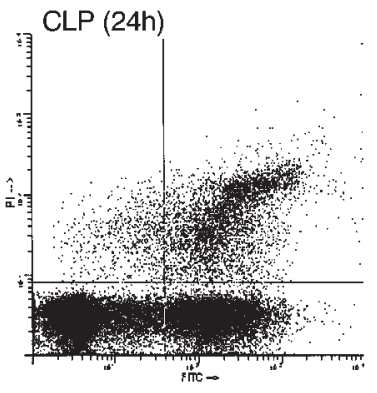

c

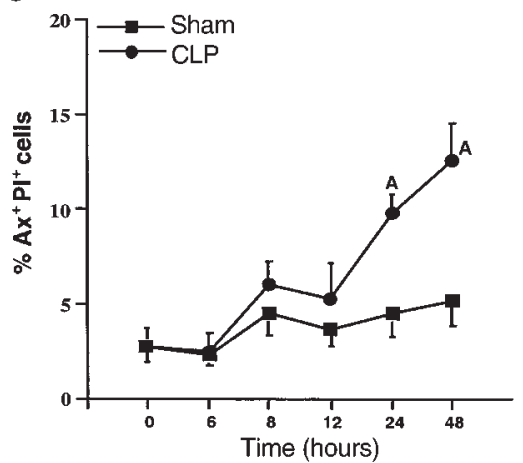

Figure 2

Flow cytometric analysis of $\mathrm{Ax}(\mathrm{FITC} \rightarrow)$ and $\mathrm{PI}(\mathrm{PI} \rightarrow)$ staining in thymocytes from sham or CLP rats. Thymocytes isolated from sham or CLP rats at the times indicated were stained with Ax-FITC and PI. (a) FACS analysis of apoptotic thymocytes from normal, sham, and CLP rats 24 hours after surgery. The regions for $\mathrm{Ax}$-positive only $\left(\mathrm{Ax}^{+} \mathrm{Pl}^{-}\right.$, lower right), $\mathrm{Pl}$-positive only $\left(\mathrm{Ax}^{-} \mathrm{Pl}^{+}\right.$, upper left), double-positive $\left(\mathrm{Ax}^{+} \mathrm{Pl}^{+}\right.$, upper right), and double-negative ( $\mathrm{Ax}^{-} \mathrm{PI}^{-}$, lower left) were established as described in Methods. (b) The percentage of early-stage apoptotic thymocytes during the course of 48 hours after sham or CLP-induced sepsis. (c) The percentage of late apoptotic and early necrotic thymocytes over 48 hours after sham or CLP-induced sepsis. ${ }^{A} P<0.05$ when compared with the sham group. ${ }^{B} P<0.01$ when compared with the sham group. Values represent means $\pm \operatorname{SEM}(n=3$ or 4 animals). 
evaluated for Bcl-2, Bcl-X $\mathrm{L}$, BAX, Bad, and Bim content 12 hours after onset of sepsis. Western blot and image analysis revealed the following: (a) Bcl-2, Bcl- $\mathrm{X}_{\mathrm{L}}, \mathrm{BAX}$, $\mathrm{Bad}$, and Bim were constitutively expressed in the thymocytes. Sham surgery had no effect on the expression of these proteins (Figure 7a). (b) However, there was a significant decrease in $\mathrm{Bcl}-\mathrm{X}_{\mathrm{L}}$ content in thymocytes from CLP rats $(P<0.05)$. Bcl- $\mathrm{X}_{\mathrm{L}}$ content was maintained at $90 \%$ of the reference control level in thymocytes from CLP rat treated with anti-C5a (Figure 7, a and $\mathrm{b}$ ). No significant changes were found in Bcl-2, $\mathrm{BAX}, \mathrm{Bad}$, and Bim content in any group (Figure $7 \mathrm{a}$ ).

$N F-\kappa B$ activation in thymocytes in sepsis; lack of effects of anti-C5a. To identify the components of translocated $\mathrm{NF}-\kappa \mathrm{B}$, thymocyte nuclear extracts from rats 12 hours after CLP were used for supershift assays with antibodies to p50, p52, p65, p68, and p75. Supershift (Figure $8 \mathrm{a}$, filled arrowhead) and an NF- $\kappa \mathrm{B}$ band of reduced intensity (Figure 8a, solid arrow) were observed with anti-p65 addition (Figure 8a). Additionally the intensity of another NF- $\kappa \mathrm{B}$ band (Figure 8a, open arrow) was reduced with anti-p50 addition. No evident supershifts were found with the addition of anti-p52, anti-p68, and anti-p75. These data suggest that the NF$\kappa \mathrm{B}$ complex in thymocytes from CLP rats was primarily composed of p50 and p65 heterodimers.

NF- $\kappa \mathrm{B}$ activation was evaluated in thymocytes 12 hours after CLP. Constitutive NF-אB p65 (Figure 8b, filled arrow) and p50 (Figure $8 \mathrm{~b}$, open arrow) were found in control rats (Figure $8 \mathrm{~b}$ ). Sham surgery did not cause NF- $\kappa$ B activation (Figure $8 \mathrm{~b}$ ). Sepsis resulted in a significant increase in nuclear translocation of NF- $\kappa B$ (Figure $8 \mathrm{~b}$ ). The strong p50 and p $65 \mathrm{NF}-\kappa \mathrm{B}$ bands were also observed in anti-C5a treatment group (Figure $8 \mathrm{~b}$ ). Image analysis revealed that the intensity of the p50 and $\mathrm{p} 65 \mathrm{NF}-\mathrm{\kappa B}$ bands was not significantly changed by anti-C5a treatment compared with CLP rats (data not shown), but instead an unknown low migrating band (open arrowhead) was enhanced. This band could not be supershifted by anti-p50, anti-p52, anti-p65, antip68, or anti-p75 (data not shown), indicating that it might be a nonspecific band.

C5a-dependent apoptosis after infusion in vivo of CVF. To explore further a relationship between C5a and thymocyte apoptosis, two experiments were carried out. The results are shown in Figure 9. In the first set of studies, $3 \mathrm{U}$ purified CVF were injected intravenously into otherwise normal rats together with $500 \mu \mathrm{g}$ preimmune IgG. Thymocytes were obtained 3 hours later. Under such conditions, plasma levels of C5a quickly peak after infusion of CVF (10). As shown in Figure 9, Ax binding to thymocytes rose from approximately $7 \%$ to $16 \%(P<0.05)$ in CVF-treated rats. Blood obtained from these animals at the 3 -hour time point failed, as expected, to show any evidence of aerobic or anaerobic bacteria (data not shown). In a companion set of rats treated with $500 \mu \mathrm{g}$ anti-C5a IgG, most of the Ax binding to thymocytes was suppressed $(9 \% ; P<0.05)$ when compared with the group treated with preimmune IgG.

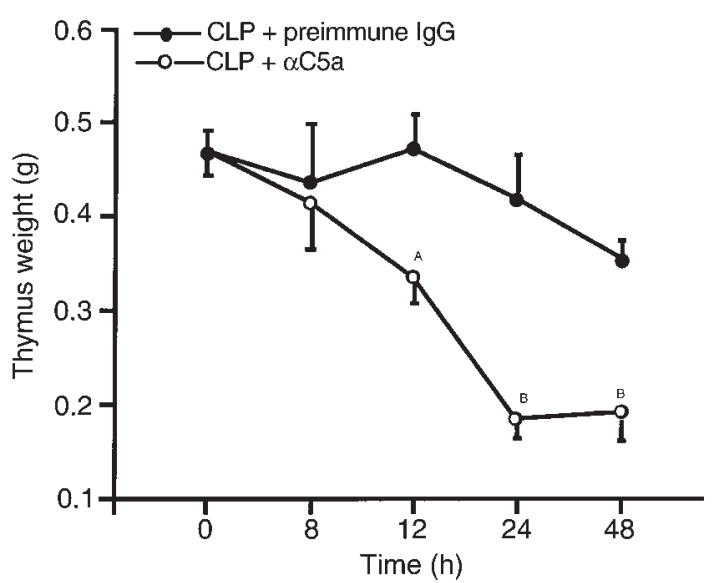

Figure 3

The effects of C5a blockade on thymus atrophy induced by CLP. Immediately after CLP surgery, animals were treated intravenously with $400 \mu \mathrm{g}$ preimmune $\operatorname{lgG}$ or $400 \mu \mathrm{g}$ anti-rat C5a $\operatorname{lgG}(\alpha C 5 \mathrm{a})$. At the indicated time points, the animals were sacrificed and the thymus was weighed. The same protocol for C5a blockade was used in all subsequent figures. For each vertical bar, $n=4$ or 5 animals. ${ }^{A} P<0.05$ when compared with IgG control. ${ }^{\mathrm{B}} P<0.01$ when compared to $\lg \mathrm{G}$ control.

Another set of rats was subjected to CLP at time 0 . Three hours thereafter, animals were infused intravenously with $3 \mathrm{U} C V F$ in the presence of $500 \mu \mathrm{g}$ preimmune IgG or $500 \mu \mathrm{g}$ anti-C5a. Three hours thereafter (or 6 hours after CLP), thymocytes were obtained and examined for Ax binding. The results are shown in Figure 9. CLP rats (at 6 hours) showed a slight increase in Ax binding (11\%; $P=$ NS when compared with controls). In CLP animals also injected at 3 hours with CVF, there was at 6 hours a substantial increase in Ax binding (to $26 \%)(P<0.05)$, the increase of which was virtually totally abolished in the presence of anti-C5a. These experiments indicate that in vivo production of C5a can induce increased Ax binding to thymocytes, a feature of apoptosis.

\section{Discussion}

Apoptosis is a mode of self-directed cell death that plays an important role in tissue homeostasis (23). It is well known that apoptosis occurs in both physiological and pathological conditions. Thymocyte apoptosis is an important process involved in T-cell maturation and selection. Negatively selected thymocytes die by apoptosis (24). In thymus, the majority of thymocytes are unselected and highly sensitive to apoptosis-inducing factors (24). It is estimated that during thymic development, more than $90 \%$ of T-cell precursors die through apoptosis $(25,26)$. In sepsis, immature thymocytes, $\mathrm{CD}^{+} \mathrm{CD}^{+}$and $\mathrm{CD}^{-}{ }^{-} \mathrm{CD} 8^{-}$cells, have been found to undergo apoptosis vigorously (8). The current study shows that markedly upregulated thymocyte apoptosis (8 hours) precedes a significant loss in thymus weight (12 hours). During the period of high-frequency apoptosis (8-24 hours), the thymus weight rapidly decreases. Apoptotic cells are recognized and phagocytized by 
a
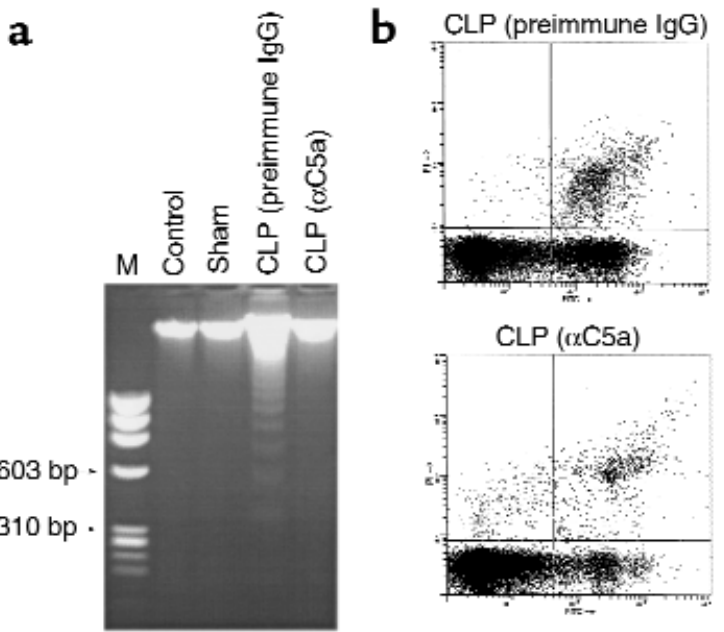

c

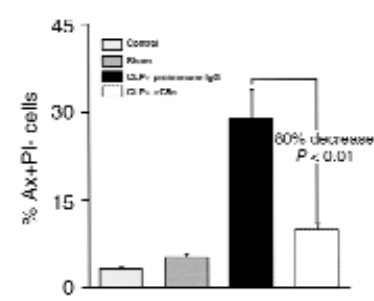

d

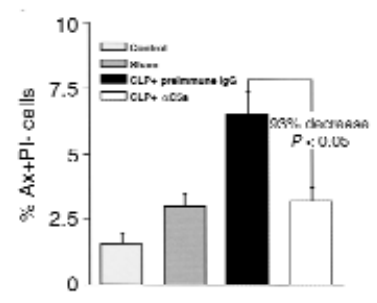

Figure 4

Protection of thymocytes from apoptosis by C5a blockade in CLPinduced sepsis. Twenty-four hours after surgery, thymocytes were harvested from control, sham, CLP-treated with preimmune IgG, and anti-C $5 \alpha$-treated CLP rats, and evaluated for DNA fragmentation and $\mathrm{Ax} / \mathrm{PI}$ staining using the same protocols described for Figures 1 and 2. (a) Effect of C5a blockade on DNA fragmentation induced by CLP, representative of three separate experiments. (b) Quantitative analysis of the effect of C5a blockade on thymocyte apoptosis by flow cytometry. The percentages of early-stage apoptotic cells (c) and late apoptotic/early necrotic cells $(\mathbf{d})$ are presented as means $\pm \operatorname{SEM}(n=4$ animals).

macrophages or adjacent cells very quickly, with the entire processing time less than 2 hours in some cells, preventing an inflammatory response $(27,28)$. This suggests that the frequency of apoptosis in this study is likely to be an underestimate. Therefore, at some time points such as 12 hours after onset of sepsis, more than $30 \%$ of the thymocytes may be undergoing apoptosis. Obviously, thymocyte apoptosis is a crucial event occurring in the thymus during sepsis, resulting in drastic reduction in thymic weight. The short-term presence of apoptotic cells and sustained thymocyte apoptosis with high frequency suggest that different phases of cells with morphological features and/or biochemical alterations exist in the thymus at various periods $(8-24$ hours) after the onset of sepsis.

The mechanism underlying thymocyte apoptosis in sepsis still remains unclear. It has been found that TNF may not be responsible for the induction of accelerated thymic apoptosis during polymicrobial sepsis (29). Blockade of corticosteriods released after the onset of

sepsis attenuates thymocyte apoptosis (29). Thymocyte apoptosis is not caused by endotoxin during experimental sepsis, although there is endotoxin-driven activation for thymic apoptosis $(30,31)$. Thymocyte apoptosis in sepsis occurs in a p53-dependent and Fas-independent pathway $(32)$. Nitric oxide $(\bullet N O)$ triggers thymocyte apoptosis but inhibits dexamethasoneinduced apoptosis, indicating that elevated levels of -NO in sepsis might play the dual role in apoptosis (33, 34). The current study shows that blockade of C5a in sepsis almost completely prevents thymocyte from apoptosis, suggesting that thymocyte apoptosis in sepsis is in some manner involved in a C5a-related pathway.

Humans or animals with sepsis display increased blood levels of proinflammatory factors: TNF- $\alpha$, INF- $\gamma$, IL-1 $\beta$, IL-6, and IL-8 $(35,36)$. Anti-inflammatory cytokines such as TGF- $\beta$, IL-4, IL-10, and IL-13 are also recruited into the vascular compartment during sepsis, apparently to counterbalance the proinflammatory responses $(35,36)$. SIRS-related cytokines such as TNF$\alpha$, IL-1 $\beta$, IL-6, and IL-10 have been implicated in apoptosis during sepsis (2). It is becoming appreciated that in sepsis, the loss of the balance between proinflammatory and anti-inflammatory mediators may result in exaggerated proinflammatory responses, the antiinflammatory response syndrome, immunosuppression, apoptosis, and organ dysfuction $(37,38)$. Elevated plasma levels of C5a have been found both in humans with sepsis and in animals with sepsis (11-14). It has been demonstrated that C5a stimulates the synthesis and release of proinflammatory cytokines such as TNF$\alpha$, IL-1 $\beta$, IL-6, and IL-8 from human leukocytes (39). An in vivo experiment reveals that the blockade of $\mathrm{C} 5 \mathrm{a}$ with

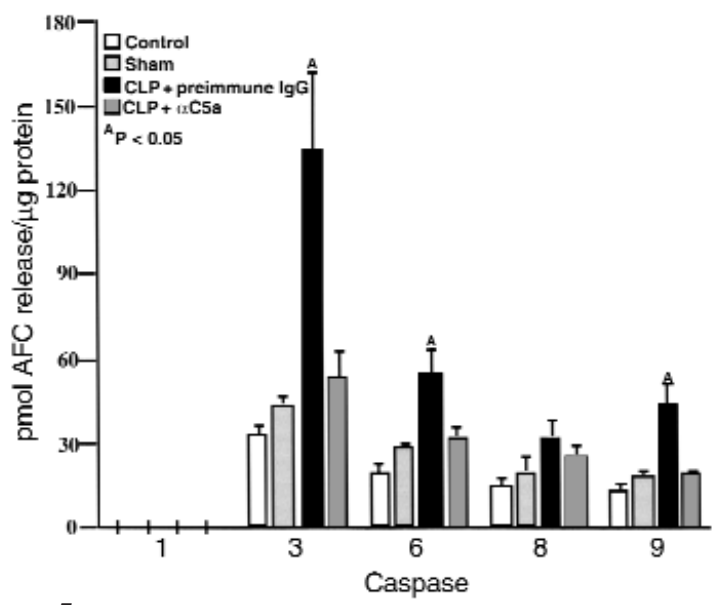

Figure 5

Inhibition of caspase activation in thymocytes by in vivo $\mathrm{C} 5 \mathrm{a}$ blockade in CLP-induced sepsis. Cell lysates were incubated with caspase substrates: Ac-YVAD-AFC (caspase-1), Ac-DEVD-AFC (caspase-3), Ac-VEID-AFC (caspase-6), Ac-IETD-AFC (caspase-8), and Ac-LEHDAFC (caspase-9). The cleaved AFC was detected on a Cytofluor II plate reader. The results were presented by picomoles of AFC released from substrates per microgram protein over a 2-hour incubation time. ${ }^{A} P<0.05$ when compared with sham or CLP $+\alpha C 5 a$ group. All values represent means $\pm \operatorname{SEM}(n=4)$. 
a Mitochondria
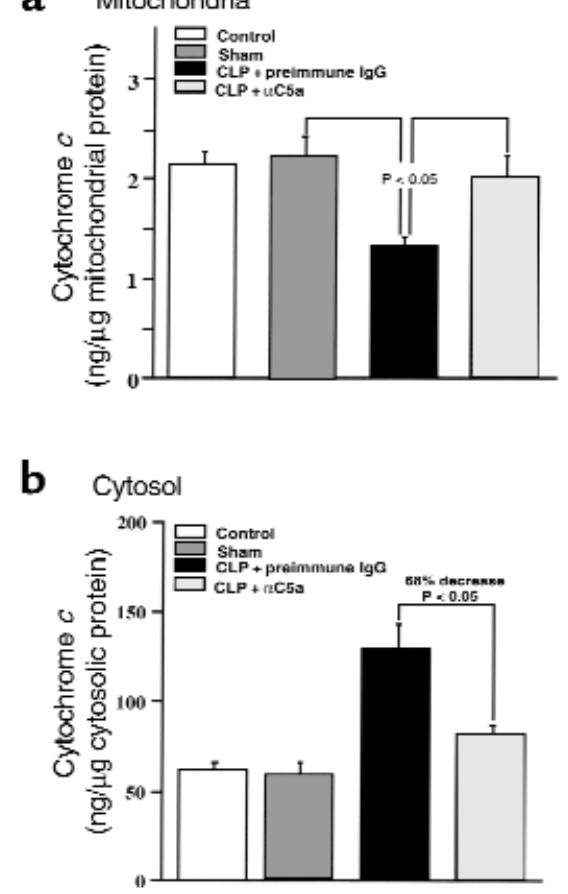

\section{Figure 6}

Effects of $\mathrm{C} 5 \mathrm{a}$ blockade on cytochrome $\mathrm{c}$ release from mitochondria in thymocytes during CLP-induced sepsis. Cytosolic and mitochondrial fractions were separated as described in methods. ( $\mathbf{a}$ and $\mathbf{b}$ ) Cytochrome $c$ concentrations in cytosol and mitochondria were quantitatively determined by ELISA. $P<0.05$ when compared with sham or $\mathrm{CLP}+\alpha \mathrm{C} 5$ a group. All values represent means $\pm \operatorname{SEM}(n=4)$.

anti-C5a in sepsis results in a decrease of over $75 \%$ in serum IL-6 bioactivity (39). These data imply that C5a might mediate cytokine expression, thereby affecting the cytokine balance. Taken together with the evidence shown in this study that C5a blockade markedly reduces thymocyte apoptosis in sepsis, it is likely that C5a blockade in sepsis results in a decrease in a group of apoptosis-inducing cytokines, thereby inhibiting apoptosis. In addition, treatment of CLP rats with antiC5a also reduces the levels of bacteremia (18). CLPinduced thymocyte apoptosis can occur in an endotoxin-independent pathway (30). In experiments designed to assess the linkage between C5a and thymocyte apoptosis, we first incubated recombinant rat $\mathrm{C} 5 \mathrm{a}$ with thymocytes obtained from normal rats and were not able to detect an increase in apoptosis (data not shown). However, in vivo infusion of CVF caused a significant increase in C5a-dependent apoptosis in otherwise normal rats or in rats in the very early stages of CLP (Figure 9). These data indicate a linkage between C5a and apoptosis. Thymocytes from normal rats appear to be resistant to the effects of C5a but may be susceptible after being exposed to a prior insult, such as CLP. Alternatively, it is possible that in vivo generation of C5a leads to enhanced release of adrenal corticosteroids, which then induce apoptosis of thymocytes. These possibilities are currently being explored. It is clear that systemic activation of complement leads to C5a-dependent thymocyte apoptosis (Figure 9). The apoptotic process can not be ascribed to induction of bacteremia, as blood cultures for aerobic and anaerobic bacteria were negative. The precise linkage between C5a and thymocyte apoptosis remains to be determined. The C5a receptor expressed on TGW neuroblastoma cells is associated with the apoptotic signal transduction pathway (40), and C5a directly causes apoptosis in TGW neuroblastoma cells (41). Moreover, it is reported that C5a receptor is expressed on the human T cells and upregulated upon phytohemagglutinin stimulation (42). Despite the mechanism behind the anti-apoptotic effect of antiC5a, C5a blockade appears to be one of the strategies to rescue thymocytes in sepsis.

Cells undergoing apoptosis are typified by morphological and biochemical features, including chromatin aggregation, nuclear and cytoplasmic condensation, blebbing of cell membrane, DNA fragmentation,

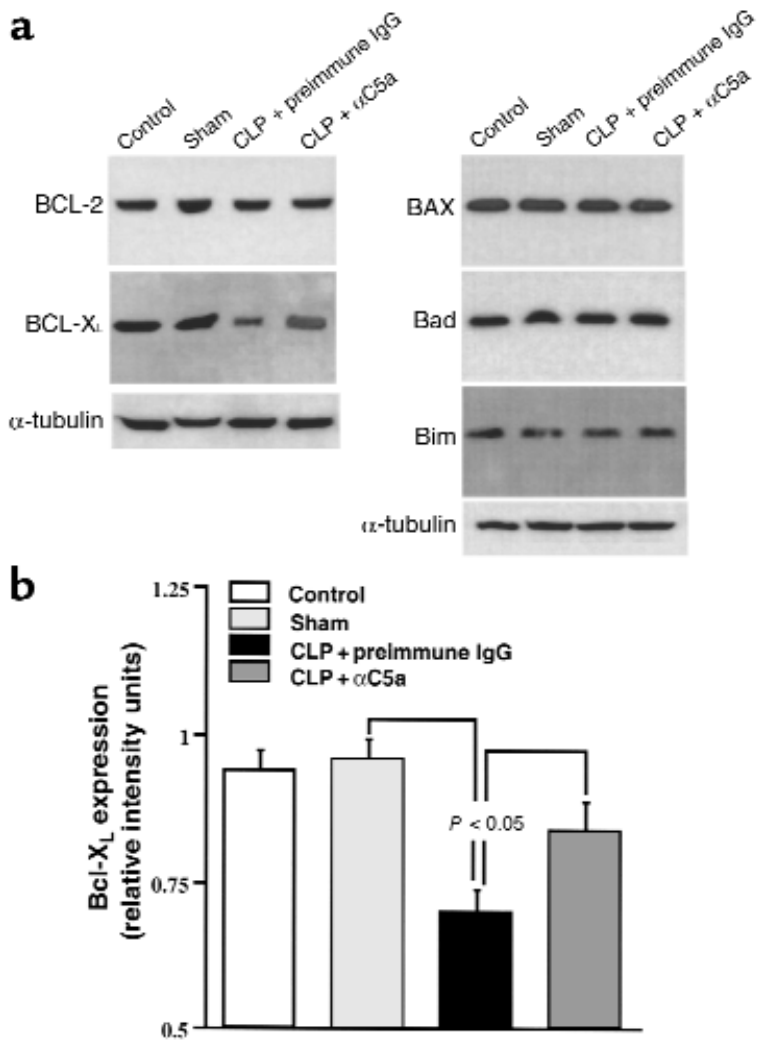

Figure 7

Effects of $\mathrm{C} 5 \mathrm{a}$ blockade on expression of Bcl-2 family members in thymocytes during CLP-induced sepsis. (a) The cytoplasmic extracts containing $100 \mu \mathrm{g}$ protein were electrophoresized in a denaturing polyacrylamide gel and then transferred to a nitrocellulose membrane. Protein expression was evaluated by Western blot using the following antibodies: polyclonal rabbit anti-Bcl-2, polyclonal rabbit anti-BAX, monoclonal mouse anti-Bcl- $X_{\mathrm{L}}$, polyclonal rabbit anti-Bad, polyclonal rabbit anti-Bim, and monoclonal mouse anti- $\alpha$-tubulin. The graphs are representative of three separate experiments. (b) Densitometric quantification of $\mathrm{BCl}-X_{\mathrm{L}}$ expression normalized to $\alpha$-tubulin. All values represent means $\pm \operatorname{SEM}(n=3)$. $P<0.05$ when compared with sham or CLP $+\alpha C 5$ a group. 


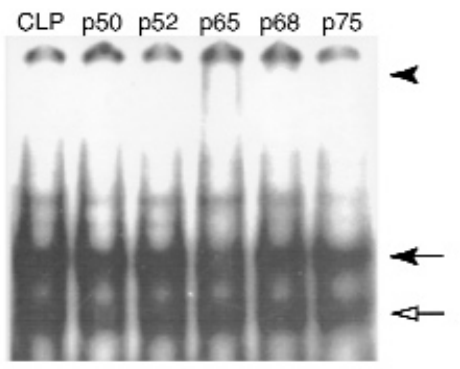

b

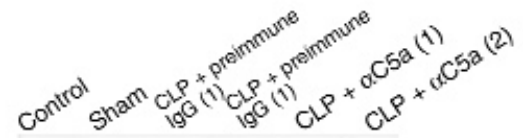

\section{Figure 8}

Effects of C5a blockade on NF- $\mathrm{KB}$ activation in thymocytes from CLP animals. (a) Supershift analysis of CLP-induced NF-KB components. DNA-binding reactions with nuclear extracts from thymocytes harvested from rats 12 hours after CLP were incubated with ${ }^{32} \mathrm{P}$-labeled $\mathrm{NF}-\kappa \mathrm{B}$ oligonucleotide in the absence or presence of antibodies to the NF- $\kappa B$ proteins indicated (p50, p52, p65, p68, p75). Supershift of p65 is indicated by a filled arrowhead. (b) Activation of NF- $\kappa B$ in sepsis and effect of C 5 a blockade. NF- $\kappa B$ activation was evaluated in thymocytes harvested from rats in various groups as indicated. Each lane represents a different animal. The filled arrow indicates

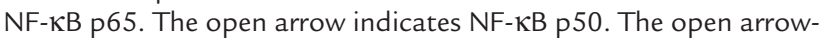
head indicates an unknown band.

release from mitochondria of cytochrome $c$ into the cytoplasm, activation of caspase cascade, and alterations in membrane asymmetry $(23,43,44)$. It is well accepted that apoptosis is regulated by intracellular signaling. Caspases, proteins of the Bcl-2 family, together with mitochondrial cytochrome $c$, have been identified as essential components of the intracellular apoptotic signaling pathways (45).

Caspases, a family of intracellular cysteine proteases, play a pivotal role in the initiation and execution of apoptosis regardless of the origin of the apoptotic stimulus $(46,47)$. Caspases are present in normal cells as inactive precursors and by cleavage become active proteases, leading to morphologic changes and cell death by enzymatically reacting with various cellular substrates $(46,47)$. Fourteen members of the caspase family have been identified and are divided into two subfamilies, initiator and executor, based on their roles in apoptotic signaling cascade (48). Caspase-3, -6 , and -7 are executors (49), whereas caspase- $2,-8,-9$, and -10 are believed to be initiators (50). In this study, elevated caspase activity after onset of sepsis was found in caspase- $3,-6$, and -9 , but not in caspase- 1 and -8 . These data are in agreement with the previous finding that caspase- $2,-3,-6$, and -9 , but not -1 , are activated in sepsis-induced thymocyte apoptosis in mice (51). Caspase3 activity elevates to a greater extent than caspase- 6 and -9 (Figure 5). These data suggest that caspase-3 but not -1 and -8 play a key role in sepsis-induced thymocyte apoptosis. Increased cytochrome $c$ in the cytosolic fraction and its concordant decrease in the mitochondrial fraction, together with elevated caspase- 9 activity, indicate that the mitochondria-dependent cytochrome c/apaf-1/caspase-9 pathway is involved in sepsisinduced thymocyte apoptosis. Cytochrome $c$ is released into the cytosol from mitochondria upon apoptotic signals and then binds to Apaf-1 and dATP. The cytochrome $c /$ Apaf- $1 /$ dATP complex recruits caspase9, leading to its activation (52). Activated caspase-9 cleaves downstream caspases such as caspase-3, -6, and -7 initiating the caspase cascade (53).

Members of the Bcl-2 protein family regulate the release of cytochrome $c$ from mitochondria and exert an anti- or proapoptotic effect during apoptosis (45). Antiapoptotic members include Bcl-2, Bcl- $\mathrm{X}_{\mathrm{L}}, \mathrm{Bcl}-\mathrm{w}, \mathrm{Mcl}-1$, $\mathrm{A} 1$, and Boo, whereas proapoptotic members are Bax, Bak, Bok, Bcl- $\mathrm{X}_{\mathrm{S}}$, Bad, Bid, Bik, Bim, Krk, and Mtd (45, 54). In the current study, we demonstrate a marked decrease in Bcl- $X_{\mathrm{L}}$ expression in thymocytes during sepsis, whereas no significant changes were found in $\mathrm{Bcl}-2$, BAX, Bad, and Bim content. Transgenic mice that selectively overexpress Bcl-2 in T cells show almost complete protection against apoptosis of $\mathrm{T}$ cells and improved

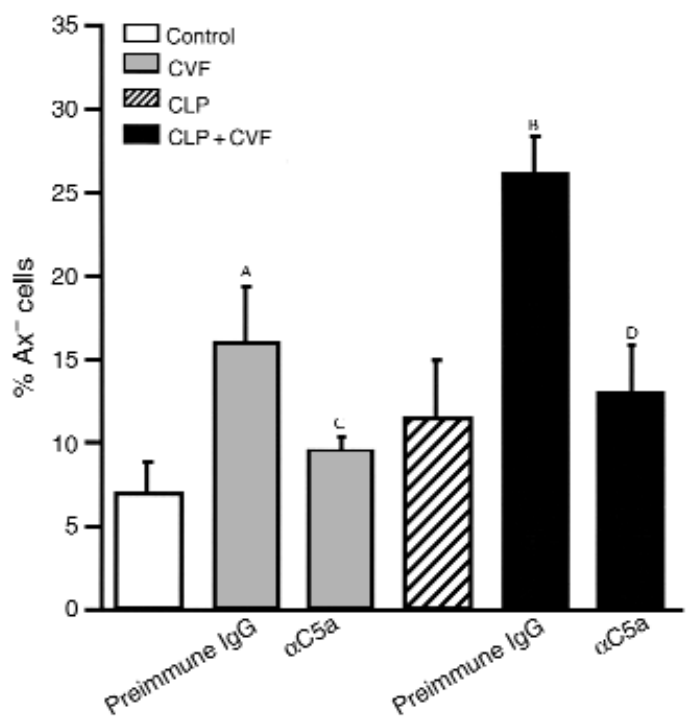

Figure 9

Effects of C5a blockade on thymocyte apoptosis induced by CVF. Rats were injected intravenously with 3 U CVF and $500 \mu \mathrm{g}$ preimmune IgG or $500 \mu \mathrm{g}$ anti-C5a, and thymocyte apoptosis was evaluated by Ax staining 3 hours after infusion. For CLP rats, CVF was given 3 hours after CLP surgery. ${ }^{A} P<0.05$ when compared with control or CVF $+\alpha C 5$ a group. ${ }^{\mathrm{B} P}<0.01$ when compared with CLP or CLP $+C V F+\alpha C 5$ a group. All values represent means \pm SEM ( $n$ $=4$ or 5$) .{ }^{C} P>0.05$ when compared with control. ${ }^{D} P>0.05$ when compared with CLP. 
survival in sepsis (9). However, no difference has been seen for Bcl-2 expression in the spleen sections from septic and nonseptic patients by means of immunohistochemistry (5). In this study, we were not able to observe a significant change in Bcl-2 expression in thymocytes obtained from sham and CLP rats by Western blots (Figure 7). This result is in conflict with the finding that shows a slight decrease of $\mathrm{Bcl}-2$ expression in thymocytes of CLP mice compared with sham mice by flow cytometry (31). This discrepancy might be caused by species differences and higher sensitivity of FACS analysis in comparison to the densitometric quantification. However Bcl- $\mathrm{X}_{\mathrm{L}}$ seems to play more important role in apoptosis in thymus than $\mathrm{Bcl}-2$. Bcl- $\mathrm{X}_{\mathrm{L}}$, like Bcl2 , interacts with $\mathrm{Bax}$ to suppress apoptosis (55). Bcl- $\mathrm{X}_{\mathrm{L}}$ protects $\mathrm{CD} 4^{+} \mathrm{CD} 8^{+}$thymocyte from apoptosis $(56,57)$, which is the major cell type in thymus (6). Bcl- $\mathrm{X}_{\mathrm{L}}$ and Bcl-2 show a reciprocal expression pattern during thymocyte maturation (26). Bcl-2 is highly expressed in $\mathrm{CD}^{-}{ }^{-} \mathrm{CD} 8^{-}, \mathrm{CD}^{+} \mathrm{CD}^{-}$, and $\mathrm{CD} 4 \mathrm{CD}^{+}$thymocytes, but is low in $\mathrm{CD}^{+} \mathrm{CD}^{+}$thymocytes. Conversely, high levels of $\mathrm{Bcl}-\mathrm{X}_{\mathrm{L}}$ are only present in $\mathrm{CD} 4^{+} \mathrm{CD} 8^{+}$, but not in $\mathrm{CD}^{-}{ }^{-} \mathrm{CD} 8^{-}, \mathrm{CD}^{+} \mathrm{CD}^{-}$, and $\mathrm{CD} 4^{-} \mathrm{CD}^{+}$thymocytes. Taken together, it is likely that the downregulated Bcl$\mathrm{X}_{\mathrm{L}}$ fails to provide a survival signal leading to immature $\mathrm{CD} 4^{+} \mathrm{CD} 8^{+}$thymocyte apoptosis in sepsis.

$\mathrm{NF}-\mathrm{\kappa B}$ transcription factors are involved in the regulation of immune and inflammatory responses and cell death (58-60). However, the role of NF- $\mathrm{KB}$ activation in apoptosis seems to be elusive. Several lines of evidence suggest a protective role of NF- $\mathrm{KB}$ activation in apoptosis, whereas others shed the lights on its proapoptotic roles (59-64). NF- $\mathrm{\kappa B}$ activation has been found in thymocytes upon various apoptosis-inducing stimuli (65). NF- $\mathrm{KB}$ induction rescues thymocytes from apoptosis induced by corticosteroids (66). Interestingly it is also reported that NF- $\mathrm{KB}$ is required for the $\alpha$ CD3-mediated apoptosis in thymocytes (67). These data suggest that NF- $\mathrm{\kappa B}$ may play different roles in thymocyte apoptosis under different microenvironment. Our data demonstrate NF- $\mathrm{KB}$ in thymocytes is activated with the components of p50 and p65 in sepsis. It seems unlikely that NF- $\mathrm{\kappa B}$ plays a proapoptotic role in thymocyte apoptosis in sepsis, given that anti-C5a almost completely inhibits thymocyte apoptosis in sepsis without affecting NF- $\mathrm{kB}$ activation (Figure 8). Further investigation is needed to define the role of NF- $\mathrm{kB}$ activation in thymocyte during sepsis.

The current study provides the first evidence to our knowledge that $\mathrm{C} 5 \mathrm{a}$ blockade in sepsis rescues thymocytes from apoptosis and provides some insights into potential pathways involved in sepsis-induced apoptosis. Our results suggest that anti-C5a treatment may inhibit thymocyte apoptosis directly or indirectly by suppressing caspase activation, preserving cytochrome $c$ in the mitochondria, and restoring $\mathrm{Bcl}-\mathrm{X}_{\mathrm{L}}$, and that anti-C5a exerts its anti-apoptotic effects via an NF$\kappa \mathrm{B}$-independent pathway in sepsis. It seems paradoxical that C5a, which is considered to be important for host defenses, should be playing a harmful role in sepsis. We believe this outcome in sepsis is due to excessive production of $\mathrm{C} 5 \mathrm{a}$, leading to "deactivation" of blood neutrophils. We have already shown that the $\mathrm{H}_{2} \mathrm{O}_{2}$ response (induced by phorbol ester) is defective (18). It seems likely that these neutrophils have lost their ability to assemble NADPH oxidase. Such possibilities are currently under study.

\section{Acknowledgments}

This study was supported by a research grant from the NIH (HL-31963). We thank Beverly Schumann and Peggy Otto for their secretarial assistance.

1. Darville, T., Giroir, B., and Jacobs, R. 1993. The systemic inflammatory response syndrome (SIRS): immunology and potential immunotherapy. Infection. 21:279-290.

2. Papathanassoglou, E.D., Moynihan, J.A., and Ackerman, M.H. 2000. Does programmed cell death (apoptosis) play a role in the development of multiple organ dysfunction in critically ill patients? A review and a theoretical framework. Crit. Care Med. 28:537-549.

3. Ayala, A., and Chaudry, I.H. 1996. Immune dysfunction in murine polymicrobial sepsis: mediators, macrophages, lymphocytes and apoptosis. Shock. 6:S27-S38.

4. Mahidhara, R., and Billiar, T.R. 2000. Apoptosis in sepsis. Crit. Care Med. 28:N105-N113.

5. Hotchkiss, R.S., et al. 1999. Apoptotic cell death in patients with sepsis, shock, and multiple organ dysfunction. Crit. Care Med. 27:1230-1251.

6. Wang, S.D., Huang, K.J., Lin, Y.S., and Lei, H.Y. 1994. Sepsis-induced apoptosis of the thymocytes in mice. J. Immunol. 152:5014-5021.

7. Barke, R.A., Roy, S., Chapin, R.B., and Charboneau, R. 1994. The role of programmed cell death (apoptosis) in thymic involution following sepsis. Arch. Surg. 129:1256-1261; discussion 1261-1262.

8. Ayala, A., Herdon, C.D., Lehman, D.L., Ayala, C.A., and Chaudry, I.H. 1996. Differential induction of apoptosis in lymphoid tissues during sepsis: variation in onset, frequency, and the nature of the mediators. Blood. 87:4261-4275.

9. Hotchkiss, R.S., et al. 1999. Prevention of lymphocyte cell death in sepsis improves survival in mice. Proc. Natl. Acad. Sci. USA. 96:14541-14546.

10. Schmid, E., et al. 1997. Neutrophil chemotactic activity and C5a following systemic activation of complement in rats. Inflammation. 21:325-333.

11. de Boer, J.P., et al. 1993. Activation of the complement system in baboons challenged with live Escherichia coli: correlation with mortality and evidence for a biphasic activation pattern. Infect. Immun. 61:4293-4301.

12. Smedegard, G., Cui, L.X., and Hugli, T.E. 1989. Endotoxin-induced shock in the rat. A role for C5a. Am. J. Pathol. 135:489-497.

13. Bengtson, A., and Heideman, M. 1988. Anaphylatoxin formation in sepsis. Arch. Surg. 123:645-649.

14. Nakae, H., et al. 1994. Serum complement levels and severity of sepsis. Res. Commun. Chem. Pathol. Pharmacol. 84:189-195.

15. Carroll, M.C., and Fischer, M.B. 1997. Complement and the immune response. Curr. Opin. Immunol. 9:64-69.

16. Kirschfink, M. 1997. Controlling the complement system in inflammation. Immunopharmacology. 38:51-62.

17. Stevens, J.H., et al. 1986. Effects of anti-C5a antibodies on the adult respiratory distress syndrome in septic primates. J. Clin. Invest. 77:1812-1816

18. Czermak, B.J., et al. 1999. Protective effects of C5a blockade in sepsis. Nat. Med. 5:788-792.

19. Morris, I.D., Lendon, R.G., Waters, C., Naylor, G., and Jones, N. 1997. Thymic regression and apoptosis in the rat after treatment with the Leydig cell cytotoxin ethylene dimethanesulphonate (EDS). Toxicology. 120:19-27.

20. Czermak, B.J., et al. 1999. In vitro and in vivo dependency of chemokine generation on C5a and TNF-alpha. J. Immunol. 162:2321-2325.

21. Nakagawa, T., et al. 2000. Caspase-12 mediates endoplasmic-reticulum-specific apoptosis and cytotoxicity by amyloid-beta. Nature. 403:98-103.

22. Lentsch, A.B., Czermak, B.J., Bless, N.M., and Ward, P.A. 1998. NFkappaB activation during IgG immune complex-induced lung injury: requirements for TNF-alpha and IL-1beta but not complement. Am. J. Pathol. 152:1327-1336. 
23. Cohen, J.J. 1993. Apoptosis. Immunol. Today. 14:126-130.

24. Cohen, J.J., Duke, R.C., Fadok, V.A., and Sellins, K.S. 1992. Apoptosis and programmed cell death in immunity. Annu. Rev. Immunol. 10:267-293.

25. Osborne, B.A. 1996. Apoptosis and the maintenance of homoeostasis in the immune system. Curr. Opin. Immunol. 8:245-254.

26. Chao, D.T., and Korsmeyer, S.J. 1997. BCL-XL-regulated apoptosis in $\mathrm{T}$ cell development. Int. Immunol. 9:1375-1384

27. Savill, J.S., et al. 1989. Macrophage phagocytosis of aging neutrophils in inflammation. Programmed cell death in the neutrophil leads to its recognition by macrophages. J. Clin. Invest. 83:865-875.

28. McCarthy, N.J., and Evan, G.I. 1998. Methods for detecting and quantifying apoptosis. Curr. Top. Dev. Biol. 36:259-278.

29. Ayala, A., et al. 1995. The induction of accelerated thymic programmed cell death during polymicrobial sepsis: control by corticosteroids but not tumor necrosis factor. Shock. 3:259-267.

30. Hiramatsu, M., Hotchkiss, R.S., Karl, I.E., and Buchman, T.G. 1997. Cecal ligation and puncture (CLP) induces apoptosis in thymus, spleen, lung, and gut by an endotoxin and TNF-independent pathway. Shock. 7:247-253.

31. Ayala, A., Xu, Y.X., Chung, C.S., and Chaudry, I.H. 1999. Does Fas ligand or endotoxin contribute to thymic apoptosis during polymicrobial sepsis? Shock. 11:211-217.

32. Hotchkiss, R.S., et al. 2000. p53-dependent and -independent pathways of apoptotic cell death in sepsis. J. Immunol. 164:3675-3680.

33. Fehsel, K., et al. 1995. Nitric oxide induces apoptosis in mouse thymocytes. J. Immunol. 155:2858-2865.

34. Ochoa, J.B., et al. 1991. Nitrogen oxide levels in patients after trauma and during sepsis. Ann. Surg. 214:621-626.

35. Wolkow, P.P. 1998. Involvement and dual effects of nitric oxide in septic shock. Inflamm. Res. 47:152-166.

36. Titheradge, M.A. 1999. Nitric oxide in septic shock. Biochim. Biophys. Acta. 1411:437-455.

37. Bone, R.C. 1996. Sir Isaac Newton, sepsis, SIRS, and CARS. Crit. Care Med. 24:1125-1128.

38. Moldawer, L.L. 1999. Organ apoptosis in the septic patient: a potential therapeutic target? Crit. Care Med. 27:1381-1382.

39. Hopken, U., et al. 1996. Inhibition of interleukin-6 synthesis in an animal model of septic shock by anti-C5a monoclonal antibodies. Eur. J. Immunol. 26:1103-1109.

40. Farkas, I., et al. 1998. A neuronal C5a receptor and an associated apoptotic signal transduction pathway. J. Physiol. (Lond.) 507:679-687.

41. Farkas, I., Baranyi, L., Liposits, Z.S., Yamamoto, T., and Okada, H 1998. Complement C5a anaphylatoxin fragment causes apoptosis in TGW neuroblastoma cells. Neuroscience. 86:903-911.

42. Nataf, S., Davoust, N., Ames, R.S., and Barnum, S.R. 1999. Human T cells express the C5a receptor and are chemoattracted to C5a. J. Immunol. 162:4018-4023.

43. Majno, G., and Joris, I. 1995. Apoptosis, oncosis, and necrosis. An overview of cell death. Am. J. Pathol. 146:3-15.

44. van Engeland, M., Nieland, L.J., Ramaekers, F.C., Schutte, B., and Reutelingsperger, C.P. 1998. Annexin V-affinity assay: a review on an apoptosis detection system based on phosphatidylserine exposure. Cytometry. 31:1-9.

45. Antonsson, B., and Martinou, J.C. 2000. The Bcl-2 protein family. Exp.
Cell Res. 256:50-57.

46. Thornberry, N.A., and Lazebnik, Y. 1998. Caspases: enemies within. Science. 281:1312-1316.

47. Thornberry, N.A. 1998. Caspases: key mediators of apoptosis. Chem. Biol. 5:R97-R103.

48. Zheng, T.S., and Flavell, R.A. 2000. Divinations and surprises: genetic analysis of caspase function in mice. Exp. Cell Res. 256:67-73.

49. Salvesen, G.S., and Dixit, V.M. 1997. Caspases: intracellular signaling by proteolysis. Cell. 91:443-446.

50. Nunez, G., Benedict, M.A., Hu, Y., and Inohara, N. 1998. Caspases: the proteases of the apoptotic pathway. Oncogene. 17:3237-3245.

51. Tinsley, K.W., et al. 2000. Caspases -2, -3, -6, and -9, but not caspase1, are activated in sepsis-induced thymocyte apoptosis. Shock. 13:1-7.

52. Kuida, K., et al. 1998. Reduced apoptosis and cytochrome c-mediated caspase activation in mice lacking caspase 9. Cell. 94:325-337.

53. Kuida, K. 2000. Caspase-9. Int. J. Biochem. Cell Biol. 32:121-124.

54. Song, Q., Kuang, Y., Dixit, V.M., and Vincenz, C. 1999. Boo, a novel negative regulator of cell death, interacts with Apaf-1. EMBO J. 18:167-178.

55. Borner, C., et al. 1994. The protein bcl-2 alpha does not require membrane attachment, but two conserved domains to suppress apoptosis. J. Cell Biol. 126:1059-1068.

56. Ma, A., et al. 1995. Bclx regulates the survival of double-positive thymocytes. Proc. Natl. Acad. Sci. USA. 92:4763-4767.

57. Grillot, D.A., Merino, R., and Nunez, G. 1995. Bcl-XL displays restricted distribution during $\mathrm{T}$ cell development and inhibits multiple forms of apoptosis but not clonal deletion in transgenic mice. J. Exp. Med. 182:1973-1983.

58. Baldwin, A.S., Jr. 1996. The NF-kappa B and I kappa B proteins: new discoveries and insights. Annu. Rev. Immunol. 14:649-683.

59. Ghosh, S., May, M.J., and Kopp, E.B. 1998. NF-kappa B and Rel proteins: evolutionarily conserved mediators of immune responses. Annu. Rev. Immunol. 16:225-260.

60. May, M.J., and Ghosh, S. 1998. Signal transduction through NFkappa B. Immunol. Today. 19:80-88.

61. Beg, A.A., and Baltimore, D. 1996. An essential role for NF-kappaB in preventing TNF-alpha-induced cell death. Science. 274:782-784

62.Jung, M., Zhang, Y., Lee, S., and Dritschilo, A. 1995. Correction of radiation sensitivity in ataxia telangiectasia cells by a truncated I kappa B-alpha. Science. 268:1619-1621.

63. Grilli, M., Pizzi, M., Memo, M., and Spano, P. 1996. Neuroprotection by aspirin and sodium salicylate through blockade of NF-kappaB activation. Science. 274:1383-1385.

64. Abbadie, C., et al. 1993. High levels of c-rel expression are associated with programmed cell death in the developing avian embryo and in bone marrow cells in vitro. Cell. 75:899-912.

65. Ivanov, V.N., and Nikolic-Zugic, J. 1997. Transcription factor activation during signal-induced apoptosis of immature CD4(+)CD8(+) thymocytes. A protective role of c-Fos. J. Biol. Chem. 272:8558-8566.

66. Wang, W., Wykrzykowska, J., Johnson, T., Sen, R., and Sen, J. 1999. A NF-kappa B/c-myc-dependent survival pathway is targeted by corticosteroids in immature thymocytes. J. Immunol. 162:314-322.

67. Hettmann, T., DiDonato, J., Karin, M., and Leiden, J.M. 1999. An essential role for nuclear factor kappaB in promoting double positive thymocyte apoptosis. J. Exp. Med. 189:145-158. 